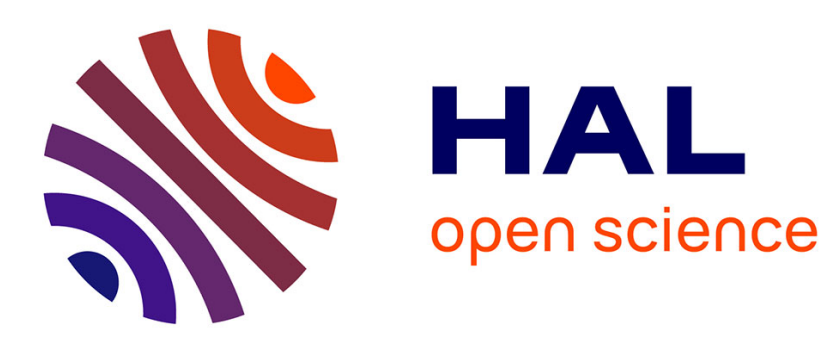

\title{
Monte Carlo Simulation of Simultaneous Ordering and Precipitation in BCC Lattice
}

\author{
M. Athènes, P. Bellon, G. Martin, F. Haider
}

\section{To cite this version:}

M. Athènes, P. Bellon, G. Martin, F. Haider. Monte Carlo Simulation of Simultaneous Ordering and Precipitation in BCC Lattice. Journal de Physique IV Proceedings, 1996, 06 (C2), pp.C2-53-C2-58. 10.1051/jp4:1996207 . jpa-00254185

\section{HAL Id: jpa-00254185 https://hal.science/jpa-00254185}

Submitted on 1 Jan 1996

HAL is a multi-disciplinary open access archive for the deposit and dissemination of scientific research documents, whether they are published or not. The documents may come from teaching and research institutions in France or abroad, or from public or private research centers.
L'archive ouverte pluridisciplinaire HAL, est destinée au dépôt et à la diffusion de documents scientifiques de niveau recherche, publiés ou non, émanant des établissements d'enseignement et de recherche français ou étrangers, des laboratoires publics ou privés. 


\title{
Monte Carlo Simulation of Simultaneous Ordering and Precipitation in BCC Lattice
}

\author{
M. Athènes, P. Bellon, G. Martin and F. Haider* \\ CE Saclay, SRMP, 91191 Gif sur Yvette cedex, France \\ * Université de Gottingen, Institut für MetallPhysik, Allemagne
}

\begin{abstract}
Fe-Al system quenched into its two-phase field undergoes a phase separation leading to the coexistence of A2 and B2 phases. Using transmission electron microscopy, three different decomposition behaviours have been reported with increasing aluminium concentration: nucleation-growth of $\mathrm{B} 2$ inside a disordered matrix, decomposition of interconnected ordered phases which was attributed to spinodal decomposition, and nucleationgrowth of A2 inside B2 domains. This work presents the first Monte Carlo simulations of simultaneous phase seperation and ordering in a two-phase A2/B2 domain. Calculations were performed using an Atomistic Kinetic Model; one vacancy allows atomic diffusion and a residence time algorithm is used. The three experimentally observed morphologies of evolution are reproduced. Moreover decomposition kinetics is compatible with the classical Lifshits-Slyosov-Wagner coarsening theory.
\end{abstract}

\section{Introduction}

Many binary alloys exhibit A2/B2 phase transitions: Fe-Si, Fe-Co, Fe-Al. The particularity of the Al-Fe phase diagram is to have a tricritical point below which both $\mathrm{A} 2$ and $\mathrm{B} 2$ coexist. A disordered solid solution quenched into this two-phase field undergoes a phase separation of both A2 and B2. Oki et al. [1] experimentally investigated $\mathrm{Fe}-\mathrm{Al}$ decomposition in this domain using transmission electron microscopy. His observations show three different decomposition behaviours with increasing Aluminium concentration: nucleation-growth of $\mathbf{B} 2$ inside a disordered matrix, decomposition of interconnected ordered phases which was attributed to spinodal decomposition, and then nucleation-growth of A2 inside B2 domains.

Phase separation has been investigated by Lifshits-Slyosov-Wagner (LSW) [2] who first predicted the coarsening kinetics of the second phase mean precipitate size. The growth kinetics of ordered domains was first studied by Lifshits-Allen-Cahn (LAC) [3, 4] and a $t^{1 / 2}$ regime was predicted instead of $t^{1 / 3}$ for coarsening.

Monte Carlo studies using the spin exchange kinetic Ising (SEKI) model in 3D yield power law regimes with exponents consistent with LSW predictions $[5,6,7]$. More recently, simulations were performed using a vacancy jump mechanism and the observed growth exponents were also in agreement with LSW prediction $[8,9]$. Other Monte Carlo simulations based on the spin flip kinetic Ising (SFKI) model both on a square lattice [10] and a cubic lattice [11] have confirmed the power law and its $1 / 2$ exponent. Other simulations using the SEKI model in 3D yield power law regimes with exponents consistent with LAC [12] predictions.

However to our knowledge, no Monte Carlo simulations were performed in order to reproduce both phase separation and ordering in a BCC lattice, such as Fe-Al in its two-phase field. Only mean field [13, 14] and phase field [15] theories were applied to the decomposition of binary systems inside a two-phase field with or without a tricritical point. These models describe the relaxation kinetics of order parameters by assuming that the rate of change of order parameter and concentration fields are proportional to the corresponding derivatives of the free energy. Using such models, it was observed that apart from a narrow strip in which decomposition occurs through usual nucleation and growth, the precipitation of the ordered phase takes place in three stages. A congruent ordering produces a transient phase followed by decomposition of the 
disordered phase at the antiphase boundaries (APB). Then coarsening due to the excess in order/disorder interfacial energy.

However, those mentioned simulations of the precipitation of an ordered phase were performed using mean field kinetics equations : they did not account for the vacancy jump diffusion mechanism, therefore any vacancy correlation effect was not treated. Furthermore, using such a theory, the absence of fluctuations prevents the study of the nucleation of the A2 phase inside ordered domains.

In this paper, we present Monte-Carlo simulations of precipitation and ordering on a BCC lattice, for a model system exhibiting A2-B2 phase transition. We have also selected the vacancy jump mechanism, since it is the one operating in real systems.

\section{Monte-Carlo Simulations.}

\subsection{Atomistic Kinetic Model}

The Monte Carlo simulations are based on the following Atomistic Kinetic Model; A rigid BCC lattice, with periodic boundary conditions and $N=L^{3}$ lattice sites $(L=64$ or 128), is considered. A binary alloy is then represented by $N_{A}$ A-atoms, $N_{B}$ B-atoms and one vacancy $\left(N=N_{A}+N_{B}+1\right)$. Interaction energies consist in pair energies between nearest and next nearest-neighbor sites: $\epsilon_{A A}^{1}, \epsilon_{B B}^{1}, \epsilon_{A B}^{1}$ between nearest-neighbor atoms and $\epsilon_{A A}^{2}, \epsilon_{B B}^{2}, \epsilon_{A B}^{2}$ between next nearest-neighbor atoms. Similarly, one defines interaction energies between an atom and the vacancy $\left(\epsilon_{A V}^{i}\right.$ and $\left.\epsilon_{B V}^{i}, i=1,2\right)$. Because the vacancy concentration is small, one supposes that the equilibrium properties of the system are not affected by the vacancy contribution to the internal energy. Diffusion of A and B atoms occur through thermally activated exchanges of an atom with the vacancy (V). According to the rate theory [16], the frequency of an A-V exchange is:

$$
\begin{gathered}
\Gamma_{A V}=\nu \exp \left\{-\frac{\Delta E_{\mathrm{AV}}^{\mathrm{act}}}{k T}\right\} \\
\Delta E_{\mathrm{AV}}^{\mathrm{act}}=E_{s}-\sum_{i \in \mathrm{nn}(\mathrm{A})} \epsilon_{A i}-\sum_{j \in \mathrm{nn}(\mathrm{V})} \epsilon_{j V}
\end{gathered}
$$

where $\nu$ is an attempt "frequency" and the activation energy $E_{A V}^{\text {act }}$ is the energy necessary to extract the A-V pair from its environment (computed in a broken-bond model) and to bring the A atom to a saddle point position where its interaction energy with the system is $E_{s}$.

Since we performed simulations at a low temperature (about $0.3 \times T_{C}$ ), we used a "residence time" algorithm $[17,18,19]$ rather than the standard Metropolis algorithm.

\subsection{Phase Diagram and interaction energies}

A model system phase diagram was constructed using the standard grand canonical algorithm. If one introduces the ordering energies, $v^{i}=\epsilon_{A A}^{i}+\epsilon_{B B}^{i}-2 \epsilon_{A B}^{i}$, with $v^{1}=0.03 \mathrm{eV}, v^{2}=-0.04 \mathrm{eV}$, then Figure 1 displays the corresponding phase diagram with coexisting $\mathrm{A} 2$ and $\mathrm{B} 2$ phases under a tricritical point. The critical temperature $T_{C}$ is set to $1175 \mathrm{~K}$ and the tricritical temperature is $T_{t r} \simeq 0.5 T_{C}$.

We introduced the asymetrical energy $u^{i}=\epsilon_{A A}^{i}-\epsilon_{A B}^{i}$ and set $u^{1}=-0.04 e V, u^{2}=0 e V$, in order to increase the mobility of the minority element and therefore to accelerate the kinetics. Although an asymetrical mobility affects the morphologies of precipitates [20], the equilibrium properties are not affected and the computation time can be significantly reduced.

\subsection{Analysis of order and concentration fields.}

Direct space : In order to visualize a local information on a site $i$, the order $\eta_{i}$ and the concentration $c_{i}$ are averaged over a cell $\Omega_{i}$ formed by the site itself, its 8 nearest neighbors, and its 6 next-nearest neighbors. 
Coefficients relating occupation sites inside $\Omega_{i}$ to $\eta_{i}$ and $c_{i}$ are calculated in such a way that B2,DO3 and B32 homogeneous phases are invariant under translation.

Reciprocal space : Decomposition kinetics can be studied using the correlation function or the structure factor $S(k)$, which is the Fourrier transform of the correlation function. Information relative to the concentration field modulations are obtained by calculating the moments centred around $k=0$. The volume fraction $f_{v}$ and the characteristic modulation length $R_{1}$ are immediately related to the two first moments: $f_{v} \propto M_{0}$ and $R_{1} \propto \frac{M_{0}}{M_{1}}$ where $M_{n}$ is defined by : $M_{n}=\sum k^{n} S(k)$.

Information relative to the order field modulations are obtained by calculating the moments centred around $k=k_{s}, k_{s}$ being the B2 superstructure vector. The long range order $S_{L R O}$ and the mean domain size $R_{2}$ are also related to the moments : $S_{L R O}=\sqrt{\frac{M_{0}}{M_{0}^{B 2}}}$ and $R_{2}=\sqrt{\frac{M_{0}}{M_{2}}}$ where $I_{B 2}=\sqrt{M_{0}^{B 2}}$ is the integrated intensity of the stochiometric B2 phase. Precipitation kinetics will be studied using the time evolution of the moments $M_{n}$ in $64^{3}$ cells.
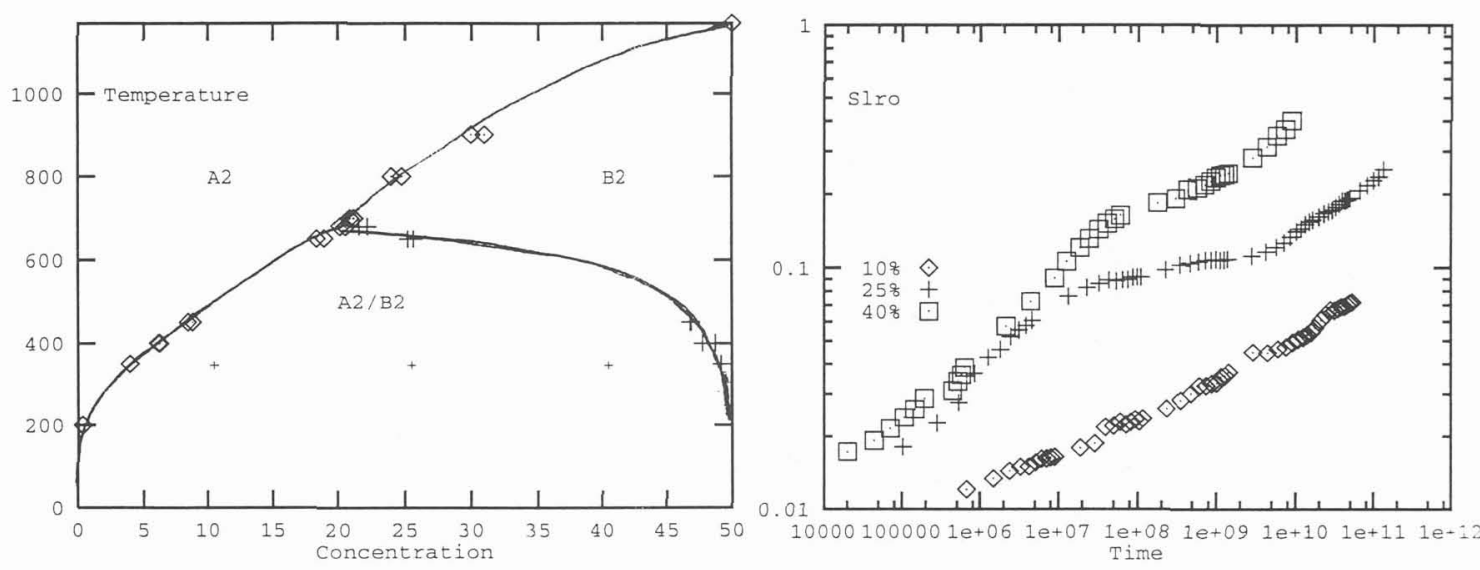

Figure 1.a: Phase diagram, 1.b Evolution of the long range order parameter (time in dimensionless units).
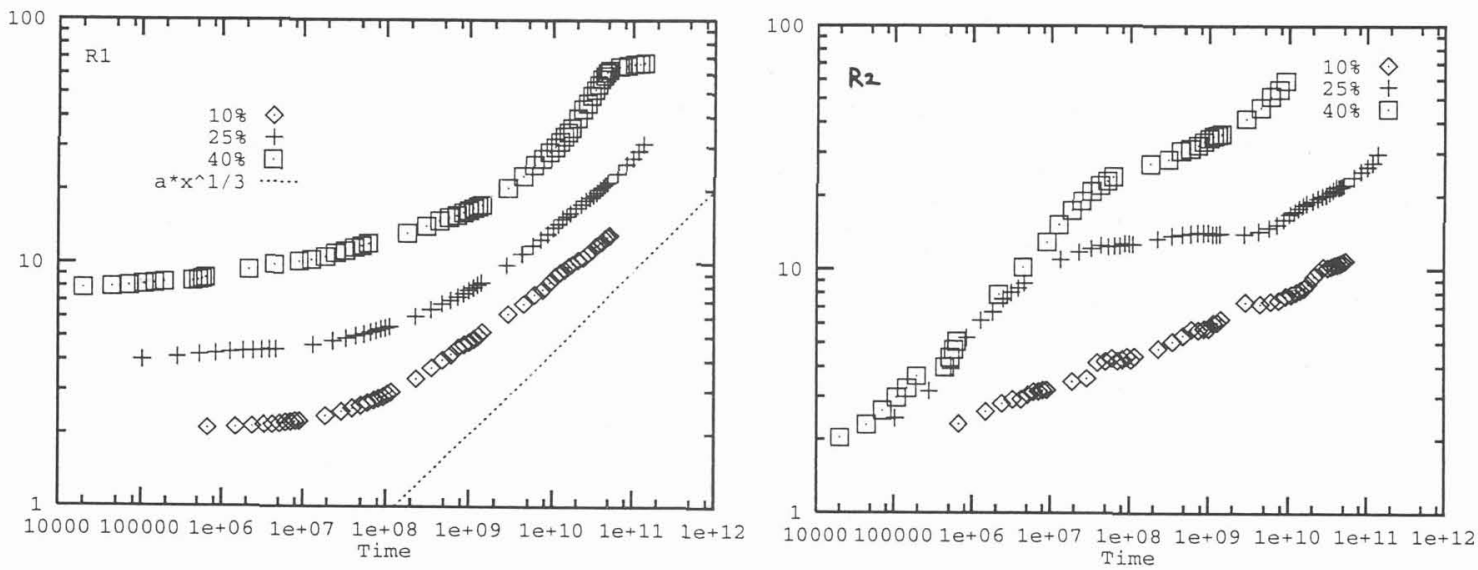

Figure 2: Evolution of (a) composition and (b) order fields. See text for definition of $R_{1}$ and $R_{2}$.

For clarity, data on Fig. 2.a have been multiplied by 0.5 and 2 for $10 \%$ and $40 \%$ respectively. 

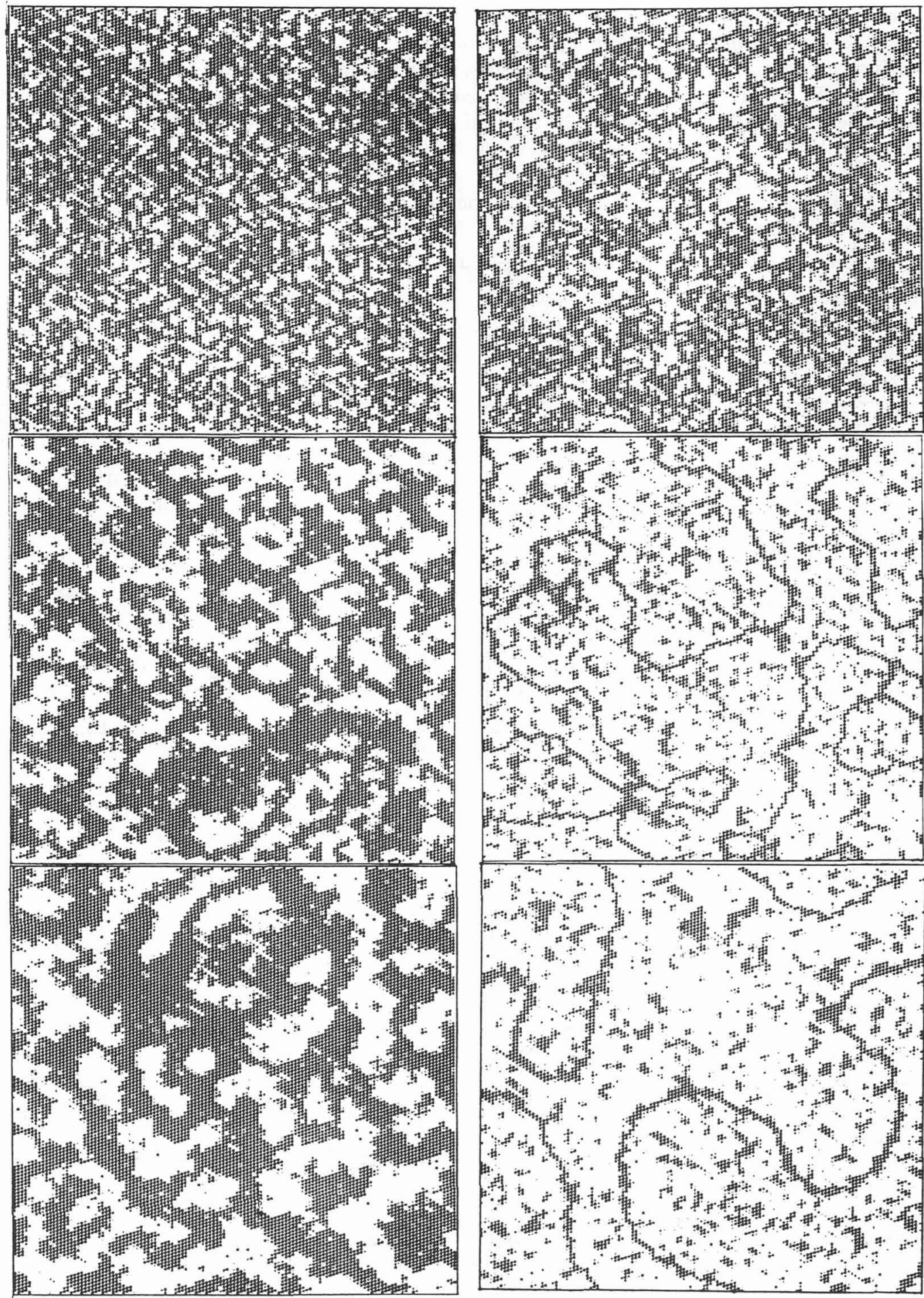

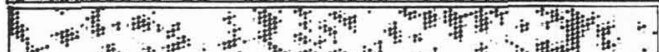

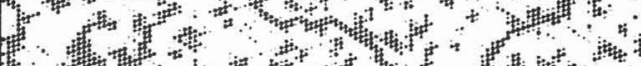
(t)

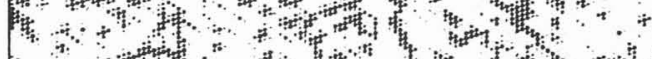

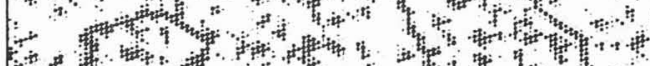

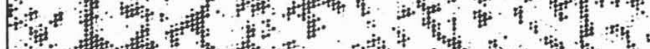
(1)

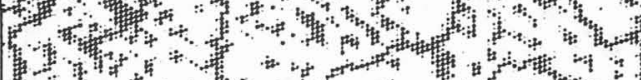
年 Ho (1)

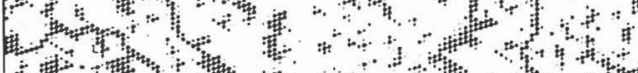
the 30

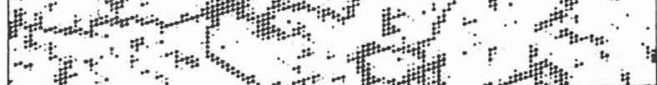

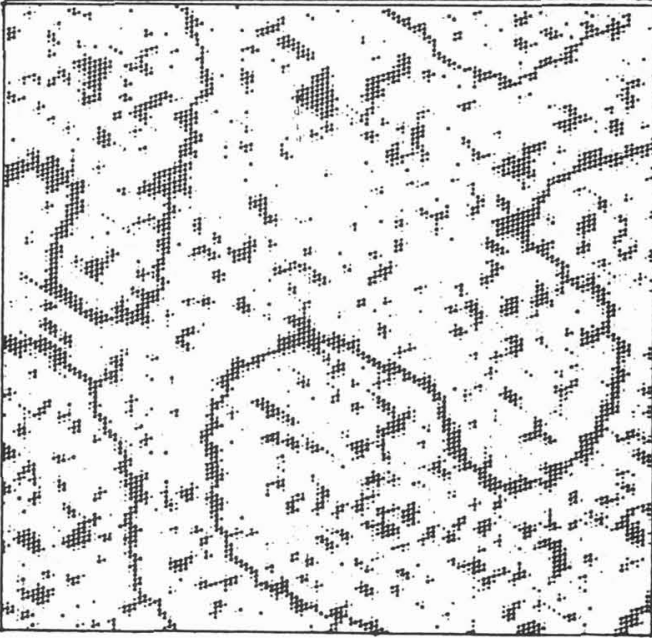

Figure 3: Ageing sequences $\left(T=348 h^{\circ}\right)$ displaying the order field. $A 2$ and $B 2$ phases are shown in dark and white respectively. Dimensionless times are (a) $1.5 .10^{8}$. (b) $1.7 .10^{10}$. (c) $4.6 .10^{10}$ for $c=25 c_{c}$, and $(\mathrm{d}) 9.10^{6}$. (e) $7.7 .10^{8}$. (f) $1.51^{10}$ for $c=40^{c} c$ 


\section{Discussions of microstructures and kinetics.}

Monte-Carlo simulations were performed at $T=348 K$ in our model system for three concentrations $c=10,25,40 \%$, which were expected to correspond to the three decomposition regimes displayed by Oki. Homogeneisation treatments were performed at $T=\infty$. Microstructures are displayed for concentrations $c=25$ and $40 \%$ only.

$10 \%$ : One observes the nucleation and the subsequent coarsening of B2 precipitates in agreement with experiments. In the reciprocal space, $S_{L R O}$ increases monotonously, while the $t^{\frac{1}{3}}$ regime for $R_{1}$ is reached after $t=10^{8}$ dimensionless units. $R_{2}$ also follows a time power law $t^{\alpha}$, with $\alpha \simeq \frac{1}{8}$.

$25 \%$ : One observes (Fig. 3.a) initial short range ordering before concentration inhomogeneities develop. At a later stage, phase seperation proceeds concomitantly with APB wetting and leads to the formation of monovariant domain inside which decomposition occurs. Pictures from (Fig. 1.c) are consistent with TEM observations although at a different scale.

The difference with the congruent long range ordering observed from TEM investigations is due to the intermediate ageing in the B2 monophase domain [1]. Therefore, we repeated Oki's experimental procedure and performed simulation after ageing at $700 \mathrm{~K}$ in $\mathrm{B} 2$ phase domain, and checked that decomposition could also develop inside an ordered domain.

The evolution of $S_{L R O}$ confirms the observed tendency (Fig. 1.b) : after an initial sharp rise, $M_{0}$ reaches a plateau, while concentration inhomogeneities begin to develop. This tendency is consistent with Oki's $\mathrm{X}$-ray diffraction measurements for samples quenched from a temperature above A2/B2 transition [21].

The fast dissolution of one of the two B2 variants results in an acceleration of $S_{L R O}$ kinetics at $t=5.10^{9}$ that stops when the computational cell does not contain any more than one variant. The assymptotic $S_{L R O}$ value corresponds to the $\mathrm{A} 2$ concentration calculated from our phase diagram. The asymptotic behaviour of $R_{1}$ is consistent with LSW power law in $t^{\frac{1}{3}}$.

$R_{2}$ evolves similarly to $S_{L R O}$. After an initial fast growth, $R_{2}$ stabilizes as decomposition decreases. One also observes the same abnormal behavior when minority variant domains shrink at $t=5.10^{9} d . u$. It results in an acceleration of the $S_{L R O}$ kinetics. Moreover, assymetry develops around the structure factor peak, which makes $R_{2}$ difficult to interpret.

$40 \%$ The same initial trend but more accentuated is observed. Short range ordering proceeds before concentration inhomogeneities develop and results in a microstructure with larger monovariant domains than at $25 \%$.

At a later stage, APB wetting proceeds and inhomogeneities inside ordered domains appear. These inhomogeneities correspond to the nuclei of the A2 phase, although it is difficult to check if A2 nuclei do not result from the dissolution of ordered domains. An intermediate ageing at $T=700 \mathrm{~K}$ before quench was performed to produce a monovariant B2 domain. This complementary simulation proved that A2 nuclei could also form inside monovariant domains; on the contrary to mean field approach, our simulated pictures reproduce the nucleation of $\mathrm{A} 2$ phase.

Calculations of the moments in the reciprocal space confirms a faster initial ordering than at $25 \%, S_{L R O}$ saturates at a higher plateau which correspond to $\frac{1}{4}$ of its maximum level. Here again, due to the instability of one variant, $S_{L R O}$ dramatically increases at $t=10^{9}$. The final $S_{L R O}$ value corresponds to the A2 volume fraction. As for ordering kinetics, phase separation is affected by the nonequilibrium of two B2 variants. The LSW regime in $t^{\frac{1}{3}}$ is reached on a small interval only, and then $R_{1}$ increases again, as variant assymetry develops. However, $R_{1}$ kinetics is not incompatible with LSW theory.

At early stages, $S_{L R O}$ growth rate increases with increasing concentration. Then, $S_{L R O}$ growth rate decreases at a value much smaller than the expected assymptotic value, which proves that after a transient fast ordering, phase seperation controls the long range ordering kinetics. 


\section{Conclusions.}

The atomistic kinetic model well reproduces the three different decomposition behaviours observed in $\mathrm{Fe}-\mathrm{Al}$ using transmission electron microscope. Moreover, in the case of a quench from $T=\infty$, the behaviour of $S_{L R O}$ and $R_{1}$ is in good agreement with Oki's diffraction measurements : after a transient fast ordering, phase seperation proceeds and long range ordering kinetics slows down. Kinetics on phase seperation enable us to fit at long times $R_{1}$ to $A+B t^{\frac{1}{3}}$ at all concentration investigated. They are therefore compatible with LSW law.

\section{References}

[1] K. Oki, S. Matsumura, T. Egushi, in Phase Transitions 10, pp 257-276 (1987)

[2] I. M. Lifshits, V. V. Slyosov, J. Phys. Chem. Solids, 19, pp 35 (1961)

[3] I. M. Lifshits, Sov. Phys. JETP, 15 pp. 935 (1962), Zh. Eksp. Teor. Phys. 42 pp. 1547 (1962)

[4] S. M. Allen, J. V. Cahn, Acta Met., 27 pp. 1085-1095 (1979)

[5] J. L. Lebowitz, J. Marro, M. H. Kalos Acta Met. 30, pp 297-310 (1982)

[6] D. A. Huse 34, N 11, pp 7845-7850 (1991)

[7] J. G. Amar, F.E. Sullivan, R. D. Mountain Phys. Rev. B 37 , pp 196 (1988)

[8] P. Fratzl, O. Penrose, Phys. Rev. B 50, N. 5, pp 3477-3481 (1994)

[9] C. Frontera, E. Vives, T. Castan, A. Planes, Submitted comment (1995)

[10] M. K. Phani, J. L. Lebowitz, J. Marro, M. H. Kalos, O. Penrose Phys. Rev Letters. 45, N 5 pp 366-369 (1980)

[11] P. S. Sahni, G. Dee, J. D.Gunton, M. K. Phani, J. L. Lebowitz, J. Marro, M. H. Kalos, Phys. Rev B, 24, N 1 pp 410-418 (1981)

[12] D. P. Landau, Physica 36-38 B, pp 731 (1977); I. Ono, Y. Matsuoka, J. Phys. Soc. Jpn. 41, pp 1425 (1976)

[13] C. Hohenberg, B. I. Halperin, Rev. Mod. Phys.49, 435, (1977)

[14] L-Q Chen, A. G. Khachaturyan, Scripta Met. 25, pp 61-66 (1991)

[15] S. Matsumura,H. Oyama and T. Egushi, K. Oki Materials Trans. , JIM 30, No. 9 (1989) 695.

[16] P. Bellon and G. Martin, Mater. Sci. Forum 155-156 (1994) 209.

[17] W. M. Young and E. Elcock, Proc. Phys. Soc. 89 (1966) 735.

[18] J.-L. Bocquet, Report CEA-R4565 (Commissariat à l"energie Atomique, Gif-sur-Yvette, 1974).

[19] A. B. Börtz, M. H. Kalos and J. L. Lebowitz, J. Comp. Phys. 17 (1975) 10.

[20] V. G. Vaks, S. V. Beiden, V. Yu Dobretsov, Pis'ma v ZhETF,61, iss. 1, pp 65-70 (1995)

[21] K. Oki, H. Sagane, T. Egushi, Japanese Jour. of Appl. Phys. 13 (1974) 753. 